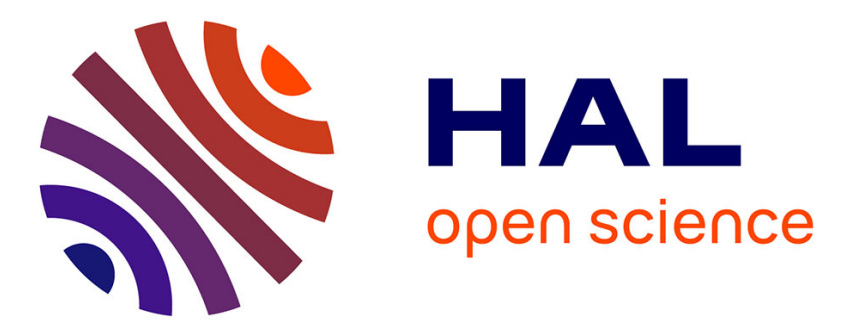

\title{
Influence of the pumping configuration on the generation of optical similaritons in optical fibers \\ Christophe Finot
}

\section{To cite this version:}

Christophe Finot. Influence of the pumping configuration on the generation of optical similaritons in optical fibers. Optics Communications, 2005, 249 (4-6), pp.553-561. 10.1016/j.optcom.2005.01.044 . hal-00474662

\section{HAL Id: hal-00474662 \\ https://hal.science/hal-00474662}

Submitted on 20 Apr 2010

HAL is a multi-disciplinary open access archive for the deposit and dissemination of scientific research documents, whether they are published or not. The documents may come from teaching and research institutions in France or abroad, or from public or private research centers.
L'archive ouverte pluridisciplinaire HAL, est destinée au dépôt et à la diffusion de documents scientifiques de niveau recherche, publiés ou non, émanant des établissements d'enseignement et de recherche français ou étrangers, des laboratoires publics ou privés. 


\title{
Influence of the pumping configuration on the generation of optical similaritons in optical fibers
}

\author{
Christophe FINOT \\ Laboratoire de Physique de l'Université de Bourgogne, Centre National de la Recherche \\ Scientifique, Unité Mixte de Recherche 5027, 21078 Dijon, FRANCE \\ christophe.finot@u-bourgogne.fr
}

\begin{abstract}
:
The impact of forward and backward amplification schemes on the generation of optical similaritons has been numerically and experimentally investigated around $1550 \mathrm{~nm}$ using a fiber based Raman amplifier. The pumping configuration does not affect the similariton nature of the output pulse, but it significantly influences the characteristics of the pulse. Shorter and higher peak-power similaritons are obtained using a backward pumping scheme. The study of the influence of the initial pulse energy also highlights the higher pumping efficiency obtained in the case of counterpropagating amplification.
\end{abstract}

\section{keywords :}

nonlinear fiber optics, ultrashort pulse amplification, parabolic pulses, amplification schemes

\section{Introduction}

The application of self-similarity techniques to the study of nonlinear pulse propagation has been the subject of much recent interest in the context of parabolic pulse generation in optical fiber amplifiers with normal group-velocity dispersion (GVD). [1-14] Such pulses, also called optical similaritons, represent a new class of solutions to the nonlinear Schrödinger equation (NLSE) with gain. They are asymptotically generated in the amplifier, independently of the shape or noise of the input pulse [1,2], and propagate selfsimilarly subject to exponential scaling of their pulse amplitude, temporal duration and spectral width [3]. In this case, the interplay of normal dispersion, nonlinearity and gain produces a linearly chirped pulse with a parabolic intensity profile [1].

Similaritons are of wide-ranging practical significance since their intrinsic resistance to the deleterious effects of the wave-breaking [4] allows the scaling of amplifiers to higherpower regimes [5,6]. Moreover, their linear chirp facilitates efficient temporal compression [5-7]. Combination of a similariton amplifier with an optical feedback has resulted in a new 
regime of laser mode-locking that is likely to have major implications for the development of high power ultrashort pulse laser oscillators [8]. Recent experimental studies have also taken advantage of the similariton characteristics to propose new methods for optical pulse synthesis [9], or for $10 \mathrm{GHz}$ telecom multiwavelength sources [10].

Those works have mainly relied on rare-earth doped fiber amplifiers with dopants such as Erbium [7,10] or Ytterbium [1,5,6,8]. However, it has been shown that a resonant amplification is not a necessary requirement for parabolic pulse generation and that Raman scattering could be efficiently used as amplification process [11]. This opens up the possibility to provide gain where no conventional amplifiers are possible.

Experimental generation of parabolic pulses have been realized with various amplification schemes : forward pumping [1,7,8,11], backward pumping [5,6] or bidirectional pumping [10]. Those different amplification schemes lead to different longitudinal gain profiles : decreasing, increasing, or nearly constant gain profiles, respectively. However, most of the numerical studies have been restricted to the case where the pulse evolution is accurately modelled by the addition of a simple constant gain term to the standard NLSE. The first theoretical work investigating the effects of an arbitrary longitudinal gain profile is the study made by Kruglov et al. [12]. In this case, the use of self-similarity techniques has revealed that the asymptotic shape of the output pulse remains a parabolic intensity profile with a positive linear chirp. The longitudinal gain profile determines only the scaling of the pulse as it propagates through the amplifier. To date, there has been no experimental confirmation of those predictions. They nonetheless remain of crucial interest for the design and optimization of high-power similariton amplifiers.

We present in this paper what is, at our knowledge, the first experimental study of the influence of the amplification scheme on the output pulse characteristics. Our amplifier at $1550 \mathrm{~nm}$ is a Raman amplifier relying on a normally dispersive Non-Zero Dispersion-Shifted Fiber (NZ-DSF) in association with a commercial watt-level CW pump source [14]. We first detail the numerical model which was used in the design of our amplifier. We then compare the results of the computations with an approximate model. The use of a frequency-resolved optical gating technique (FROG) [15] allows us to carry out a detailed study of the dependence of the output pulse characteristics on the configuration scheme and pulse energy. Finally, the experimental results are quantitatively compared with the results of the numerical model. 


\section{Numerical simulations}

Let us first present numerical simulations that demonstrate the influence of the amplification configuration under typical experimental conditions. Our simulations are based on the generalized extended NLSE [16] that rigorously includes the Raman amplification process through an appropriate integral term, as well as effects such as higher-order dispersion terms and self-steepening :

$$
\begin{aligned}
& i \frac{\partial \psi}{\partial z}=-i \frac{\alpha}{2} \psi+\frac{\beta_{2}}{2} \frac{\partial^{2} \psi}{\partial t^{2}}+i \frac{\beta_{3}}{6} \frac{\partial^{3} \psi}{\partial t^{3}} \\
& -\gamma\left[1+\frac{i}{\omega_{s}} \frac{\partial}{\partial t}\right] \psi(z, t) \int_{0}^{\infty} R\left(t^{\prime}\right)\left|\psi\left(z, t-t^{\prime}\right)\right|^{2} d t^{\prime}
\end{aligned}
$$

The function $R(t)=\left(1-f_{r}\right) \delta(t)+f_{r} h_{r}(t)$ includes the instantaneous electronic response and the delayed Raman contribution $h_{r}(t)$, with the fractional Raman contribution $f_{r}$ $=0.18$. For $h_{r}(t)$, we used the measured Raman response of fused silica [17].

The amplifier considered in this work is based on a commercial NZ-DSF. The fiber length is $L=5.3 \mathrm{~km}$ and the dispersion parameters at $1550 \mathrm{~nm}$ yields a GVD parameter $\beta_{2}=$ $4.610^{-3} \mathrm{ps}^{2} \cdot \mathrm{m}^{-1}$, and a third order dispersion $\beta_{3}=1.010^{-6} \mathrm{ps}^{3} \cdot \mathrm{m}^{-1}$, so that the fiber is normally dispersive at the wavelength of the input pulses into the amplifier. The nonlinearity coefficient is $\gamma=2.010^{-3} \mathrm{~W}^{-1} \cdot \mathrm{m}^{-1}$. The wavelength dependent loss coefficient $\alpha$ is also included in Eq. (1).

Here, $\psi(z, t)=\psi_{s}(z, t)+\psi_{p}(z, t) \exp (-i \Omega t)$, where $\psi_{s}$ and $\psi_{p}$ are the slowly varying envelopes of signal and pump fields oscillating at $\omega_{s}$ and $\omega_{p}$, respectively. $\Omega=\omega_{p}-\omega_{s}$ is the pump-signal frequency detuning chosen so that near optimal Raman gain for the signal is achieved at $1550 \mathrm{~nm}$. The input pulses $\psi_{s}(0, t)$ correspond to chirp-free Gaussian pulses with a full width at half maximum (FWHM) temporal width of $10 \mathrm{ps,}$ and an initial energy of $3 \mathrm{pJ}$. In the second part of this paper, we will also consider pulses with an initial energy of $1.5 \mathrm{pJ}$. The pump is assumed to be a noise free continuous wave at $1455 \mathrm{~nm}$. Its power is adjusted in order to provide a total integrated gain of $20 \mathrm{~dB}$ for the signal. This corresponds, in the case of a counterpropagating configuration, to a power $\left|\psi_{p}(L)\right|^{2}$ of $1.15 \mathrm{~W}$ and in the case of a copropagating scheme, to a power $\left|\psi_{p}(0)\right|^{2}$ of $1.22 \mathrm{~W}$.

In order to improve the computation effectiveness, after checking the absence of any new spectral components generated by four wave mixing or higher-order Raman effect, we use, in the case of a copropagating amplification, the following set of two coupled extended 
NLSE's (2a) and (2b) [16], with the subscripts $s$ and $p$ indicating the signal and pump wavelengths respectively. The walk-off parameter is defined as $\delta=\beta_{l p}-\beta_{l s}$ where $\beta_{1}=d \beta$ $/ d \omega$ (where $\beta$ the propagation constant).

$$
\begin{array}{r}
i \frac{\partial \psi_{s}}{\partial z}=-i \frac{\alpha_{s}}{2} \psi_{s}+\frac{\beta_{s 2}}{2} \frac{\partial^{2} \psi_{s}}{\partial t^{2}}+i \frac{\beta_{s 3}}{6} \frac{\partial^{3} \psi_{s}}{\partial t^{3}} \\
-\gamma_{s}\left[1+\frac{i}{\omega_{s}} \frac{\partial}{\partial t}\right]\left\{\left(1-f_{r}\right) \psi_{s}\left(\left|\psi_{s}\right|^{2}+2\left|\psi_{p}\right|^{2}\right)\right. \\
+f_{r} \psi_{s} \int_{0}^{\infty} h\left(t^{\prime}\right)\left(\left|\psi_{s}\left(z, t-t^{\prime}\right)\right|^{2}+\left|\psi_{p}\left(z, t-t^{\prime}\right)\right|^{2}\right) d t^{\prime} \\
\left.+f_{r} \psi_{p} \int_{0}^{\infty} h\left(t^{\prime}\right) \psi_{s}\left(z, t-t^{\prime}\right) \psi_{p}^{*}\left(z, t-t^{\prime}\right) e^{-i \Omega t} d t^{\prime}\right\} \\
i \frac{\partial \psi_{p}}{\partial z}=-i \frac{\alpha_{p}}{2} \psi_{p}-i \delta \frac{\partial \psi_{p}}{\partial t}+\frac{\beta_{p 2}}{2} \frac{\partial^{2} \psi_{p}}{\partial t^{2}}+i \frac{\beta_{p 3}}{6} \frac{\partial^{3} \psi_{p}}{\partial t^{3}} \\
-\gamma_{p}\left[1+\frac{i}{\omega_{p}} \frac{\partial}{\partial t}\right]\left\{\left(1-f_{r}\right) \psi_{p}\left(\left|\psi_{p}\right|^{2}+2\left|\psi_{s}\right|^{2}\right)\right. \\
+f_{r} \psi_{p} \int_{0}^{\infty} h\left(t^{\prime}\right)\left(\left|\psi_{s}\left(z, t-t^{\prime}\right)\right|^{2}+\left|\psi_{p}\left(z, t-t^{\prime}\right)\right|^{2}\right) d t^{\prime} \\
\left.+f_{r} \psi_{s} \int_{0}^{\infty} h\left(t^{\prime}\right) \psi_{p}\left(z, t-t^{\prime}\right) \quad \psi_{s}^{*}\left(z, t-t^{\prime}\right) e^{i \Omega t} d t^{\prime}\right\}
\end{array}
$$

In the case of a backward pumping, we also use Eq. (2a) to model the signal amplification. As regards to the pump evolution, we use an equation similar to Eq. (2b) and make some further approximations. As it will be highlighted in the next section, pump depletion effects play a minor role for the range of initial signal energies tested here in the backward configuration. We can then neglect pump depletion consequences on the temporal intensity profile of the pump. On the other hand, the influence of pump depletion on the longitudinal pump power evolution will be taken into account by the introduction of a term $\alpha_{d}(z)$, which can be numerically determined by an iterative process based on an average power analysis [18]. It can also be shown that cross non-linear terms play a negligible role and that the nonlinearity induced by the pump itself can be removed in Eq. (2b) as it leads to a constant phase shift. Based on all those approximations, an initial continuous pump will remain continuous during its propagation in the amplifier, so that we can remove in Eq. (2b) the time-dependent terms. By transforming $z$ into $-z$ in order to model the backward propagation direction of the pump, Eq. (2b) reduces to : 


$$
\frac{\partial \psi_{p}}{\partial z}=\frac{\alpha_{p}+\alpha_{d}(z)}{2} \psi_{p}
$$

To solve Eqs.(2), we use the standard split-step Fourier method [16] . For both pumping configurations tested, with the above described parameters, after a few kilometres of propagation, the output signal pulses exhibit the characteristics of a parabolic pulse. In other words, the signal can be fit by means of a parabolic intensity profile with a linear positive chirp, corresponding to an electric field of the form :

$$
\begin{gathered}
\psi_{s}(t)=A_{P}\left[1-\left(\sqrt{2} t / T_{p}\right)^{2}\right]^{1 / 2} \exp \left[i\left(\varphi_{o}-\frac{C_{P}}{2} t^{2}\right)\right],|t| \leq T_{p} / \sqrt{2} \\
\psi_{s}(t)=0,|t| \geq T_{p} / \sqrt{2}
\end{gathered}
$$

where $T_{P}$ is the FWHM temporal width, $C_{P}$ is the chirp parameter, $\varphi_{0}$ is an arbitrary phase offset and $A_{P}$ is the pulse amplitude.

In figure 1(a) we plot the evolution of the FWHM temporal width of the signal pulse (circles) according to the propagation distance $z$ for counterpropagating and copropagating pumping configurations (curves A and B respectively). We can clearly see from Fig. 1 the influence of the pumping configuration : in configuration $\mathrm{A}$, the output pulse temporal width is $43 \mathrm{ps}$, whereas in the other configuration, the amplification leads to a 52 ps pulse. As the output pulses are of the same energy, the peak powers also vary proportionally.

We represent on Fig. 1(b) the longitudinal evolution of the gain $g(z)=(\partial / \partial z)(\ln ($ $\left.\left|\psi_{s}(z)\right|^{2} /\left|\psi_{s}(0)\right|^{2}\right)$ ). Evolutions for counter- and co- propagating pumping scheme (curves A and B) are in good agreement with exponential fits $g_{f}(z)$ (an increasing and decreasing exponential $: g_{f}(z)=0.6910^{-3} e^{8.29} 10^{-5} z$ and $g_{f}(z)=1.1210^{-3} e^{-1.1610^{-4} z}$ for curves $\mathrm{A}$ and $\mathrm{B}$, respectively). For comparison, curve $\mathrm{C}$ is obtained in the case of a constant gain $g=0.56$ $10^{-3} \mathrm{~m}^{-1}$ configuration which leads to a $20 \mathrm{~dB}$ amplification.

The results given by Eqs. (2) are further compared with the predictions based on a more simple model which relies on the NLSE associated with the longitudinally varying gain $g(z)$ computed above :

$$
i \frac{\partial \psi_{s}}{\partial z}=i \frac{g(z)}{2} \psi_{s}+\frac{\beta_{2}}{2} \frac{\partial^{2} \psi_{s}}{\partial t^{2}}-\gamma\left|\psi_{s}\right|^{2} \psi_{s}
$$


Results based on the numerical integration of Eq. (4) are plotted Fig. 1a by solid lines, dots and dashed lines for A, B, C configurations respectively.

Kruglov et al. [12] have shown that Eq.(4) admits, in the asymptotic limit, a similariton solution whose FWHM temporal width $T_{P}$ depends on the gain profile $g(z)$ through the following differential equation :

$$
\frac{d^{2} T_{P}}{d z^{2}}=3 \sqrt{2} \frac{\beta_{2} \gamma}{T_{P}^{2}} U_{o} \exp \left[\int_{z_{o}}^{z} g\left(z^{\prime}\right) d z^{\prime}\right],
$$

subject to the additional boundary condition : $\left.d T_{P} / d z\right)_{z_{o}}=-\beta_{2} C_{P o} T_{P_{o}}$ where $T_{P o}, C_{P o}$ and $U_{o}$ correspond respectively to the values of the temporal duration, linear chirp and energy of the pulse at a distance $z_{o}$ such that the amplified pulse has already entered into the parabolic pulse amplification regime. The predictions given by (5) are represented Fig. 1.(a) by diamonds.

It is clearly seen from Fig. 1.(a) that for the amplifier parameters considered here, the three different models lead to similar evolutions. As Equations (4) and (5) are not specific to the Raman amplification process, this outlines that the effects described in this paper are not linked to the nature of the amplification process. In other words, the conclusions of our numerical and experimental works are not restricted to Raman amplifiers and can also be extended to amplification in rare-earth doped fibers, as long as the pulse spectral width is less than the amplifier bandwidth.

For the study of the Raman amplifier described in this paper, we may note that the model based on Eqs (2) is the only one which takes into account the temporal depletion effects of the Raman pump [14] and which includes the frequency dependence of the Raman gain. Moreover, Eqs (2) do not require an a-priori knowledge of the gain evolution along the fiber. Let us also note that Eq. (5) is only valid when the pulse enters its asymptotic evolution at a distance $z_{o}$ and, in order to have access to the parameters $T_{P o}$ and $C_{P o}$ a numerical approach is still needed.

In the remainder of this paper, we only consider numerical integrations of the two coupled NSLE's (2). 


\section{Experimental results}

\subsection{Experimental set-up}

Figures 2(a) and 2(b) show schematic representations of the experimental setups used for co- and counter-propagating amplification schemes, respectively. The $1550 \mathrm{~nm}$ input pulses were obtained from a Pritel FFL passively mode-locked fibre laser. The repetition rate was $22 \mathrm{MHz}$ and the initial pulses had a nearly Fourier transform-limited intensity profile with a temporal FWHM width of 10.2 ps. The energy of the pulses can be adjusted : in our experiments, we used pulses with energy in the range of $0.5-4.5 \mathrm{pJ}$.

The Raman gain was provided by a CW Keopsys 2W Raman laser working at 1455 $\mathrm{nm}$. The pump power was experimentally adjusted to achieve an integrated gain of $20 \mathrm{~dB}$. A WDM coupler with high power rating enabled pump and signal beams to be superposed in 5.3 $\mathrm{km}$ of the NZ-DSF whose parameters have been reported in the previous section. In the backward configuration case, an optical isolator was inserted in order to protect the picosecond laser from the residual pump wave.

Input and output pulses were characterized by a FROG device based on second harmonic generation [15]. The phase and intensity retrieval was performed using the generalized projection algorithm. The fidelity of the FROG measurements was checked using standard techniques based on comparisons of the independently-measured autocorrelation and spectrum with the FROG trace marginals.

\subsection{Output pulses}

Figures 3(a) and 3(b) show the intensity and chirp as retrieved from FROG measurements, for the two initial pulse energies of 1.5 and $3 \mathrm{pJ}$ respectively. The input intensity profile is represented in Fig. 3(a) by diamonds. Output pulses generated in forward and backward pumping configuration are displayed by crosses and open circles, respectively.

In Fig 3.(a), the initial pulse energy is 1.5 pJ. The output pulses exhibit parabolic intensity profiles with peak power of $4 \mathrm{~W}$ and $4.9 \mathrm{~W}$, associated with FWHM temporal width of 40.5 ps and 34 ps for co- and counter-propagating configurations, respectively. In Fig. 3.(b), which corresponds to $3 \mathrm{pJ}$ initial pulse energy, the peak power becomes $6 \mathrm{~W}$ and $8 \mathrm{~W}$ (associated with duration of 50.1 and $41.6 \mathrm{ps)}$ for co- and counter-propagating configurations, respectively. 
For both pulse energies, the pulses exhibit similariton features, ie, a parabolic intensity profile with a linear positive chirp. The shortest pulses with the highest peak power are obtained in the backward configuration.

In Fig (3), we compare the experimental data with numerical results (solid and dashed lines for counter- and co-propagating configurations) based on the numerical integration of Eqs. (2), with $\psi_{s}(0, t)$ given by the FROG characterization of the initial pulse. Let us note that a very good agreement between numerical and experimental results is obtained.

We may remark a small asymmetry in the intensity profile of the amplified pulses, which can be in part explained by the asymmetry of the initial pulse. But numerical simulations carried out with symmetric input pulses reveal that an asymmetry of the output pulses can also be observed in that case. That means that other phenomena occur such as third order dispersion, the spectral asymmetry of the Raman gain or the self-steepening. In the a forward configuration, pump depletion associated with walk-off effects is the main origin of this asymmetry, since the leading edge of the pulse experiences more gain than the trailing edge.

\subsection{Influence of the initial pulse energy}

Additional experiments were carried out to study the influence of the initial pulse energy on the amplified temporal width and on the pump required to achieve a $20 \mathrm{~dB}$ amplification. We considered initial pulses with energies in the range $0.5-4 \mathrm{pJ}$, and we have measured the FWHM temporal width of the autocorrelation function of the output pulses. Results are shown in Fig 4(a) with crosses for the copropagating amplification scheme and circles for the counterpropagating scheme. For each measurement, the pump power injected in the NZ-DSF was adapted in order to obtain a $20 \mathrm{~dB}$ integrated gain. The evolution of the input pump power versus the initial pulse energy is represented in Fig 4(b).

The evolution of the temporal width versus the initial energy clearly emphasizes the intrinsic non-linear behaviour of the similariton generation : when the initial pulse energy increases from $0.5 \mathrm{pJ}$ to $4.5 \mathrm{pJ}$, we observe an increase of $66 \%$ and $100 \%$ of the duration of the output pulse for a counter- and co-propagating amplification scheme, respectively. This evolution is found to be in good agreement with the numerical model based on Eqs. (2). The case of a constant longitudinal gain is also shown by the dashed line : in this case, the 
evolution of the temporal width relative to the input pulse energy is intermediate between the two previous cases.

We may note in Fig. 4(b) that forward and backward amplification schemes (crosses and circles) lead to different pump power evolutions with respect to the initial pulse energy. For a co-propagating pump, the pump power has to be increased with the input signal energy : the pump power required to achieve a $20 \mathrm{~dB}$ amplification of a $4 \mathrm{pJ}$ initial pulse energy is $1.37 \mathrm{~W}$ which is $8 \%$ higher than the pump power required in the case of a $0.5 \mathrm{pJ}$ initial pulse energy. In the counterpropagating configuration, for this range of pulse energies and for low repetition rates, we do not experimentally observe such a dependence of the initial pulse energy on the pump power needed to achieve a fixed amplification.

In forward and backward configurations, the longitudinal evolution of the gain is affected by the attenuation of the pump due to linear losses within the fiber. Nevertheless, as the linear losses are independent of the initial pulse energy, pump losses cannot explain the dependence experimentally observed in the case of a forward pumping. The pump evolution is also affected by its depletion owing to energy transfer from the pump to the signal. Such pump depletion will act very differently depending on the amplification scheme. When pulses and pump copropagate, the temporal pump depletion effects are localized : this may have significant effects when the signal peak power exceeds that of the pump intensity[14]. As a consequence of this severe localized depletion, pump power has to be increased whenever the initial pulse energy grows larger.

When pulse and pump counterpropagate, no such localized depletion exists : due to the high group velocity mismatch of pump and signal within the fiber (nearly twice the speed of light in the fiber), interactions between signal and pump are not temporally localized. In this case, one must only take into account the average power of the amplified pulses. For the parameters used in our experiments, the average output power was less than $10 \mathrm{~mW}$, which is only $1 \%$ of the pump power used. Depletion effects of the pump can then be neglected so that the evolution of the required pump power for a $20 \mathrm{~dB}$ amplification does not depend on the initial pulse energy.

In the case of forward pumping, we would like to outline that the walk-off effects between the pump and the signal can reduce the influence of the localized pump depletion. As the signal and pump propagate with slightly different velocities, the continuous temporal shift of the pump wave with respect to the temporal position of the signal pulse leads to an artificial regeneration of the pump as seen by the pulse [14]. In the absence of walk-off effects, it 
would not have been possible to generate output pulses with a peak power four times higher than the pump intensity. However, the limited value of the walk-off parameter ( $\delta=0.65$ ps. $\mathrm{m}^{-1}$ in the copropagating scheme) is not sufficient to completely suppress the temporal effects of pump depletion.

Let us finally compare the experimental evolution of the pump power needed to achieve a $20 \mathrm{~dB}$ amplification versus the initial energy with the results predicted by the model developped in Eq. (2). We can see in Fig. 4.(b) that the global evolutions for experimental and theoretical cases are the same. But the experimental values of the pump power required to achieve a $20 \mathrm{~dB}$ amplification are higher than the predictions of the numerical simulation by 9 $\%$. This difference can be explained with the help of polarization considerations. Indeed, whereas the signal pulses are linearly polarized, the pump polarization is scrambled, so that the effective power acting in the amplification process is lower. Moreover, as the spectral width of the pulse is more than $0.5 \mathrm{THz}$ and the propagation length is $5.3 \mathrm{~km}$, polarization mode dispersion (PMD) effects in Raman fiber-based amplifier can affect the propagation.

The pump power reduction factor of $9 \%$ between experiment and numerical simulations is in qualitative agreement with the reduction of the nonlinear parameter by a factor of $8 / 9$ which is commonly used in the case of random birefringence [16]. However, some further experiments would be needed to conclude to the validity of the theoretical model which predicts a reduction of the non-linear parameter by a factor 8/9. A recently developed vectorial formalism which includes the PMD effects in Raman fiber-based amplifiers could be used to consider these aspects in more detail [19] but is beyond the scope of this paper.

\section{Conclusion.}

We compared in this paper co- and counter- propagative pumping schemes for a Raman amplifier and we have demonstrate numerically and experimentally the significant impact of the choice of the amplification scheme. The pumping configuration does not affect the global shape of the amplified pulse, but it significantly influences the characteristics of the similariton pulse. Shorter and higher peak-power similariton pulses are obtained when using a backward pumping scheme. Another advantage of the backward configuration is the absence of the localized temporal depletion effects, which leads to a higher pumping efficiency. Numerical simulations based on a set of coupled NSLE accurately model all those features. 
As the results of this paper are not restricted to the Raman amplification process and can be extended to rare-earth doped amplifiers, our conclusions can give practical guidelines to the realization of high-power short-pulse amplifiers when the highest signal to noise ratio is not the main constraint.

In the context of ultrashort pulse generation, we can note that similaritons develop a higher spectral width in the forward configuration so that shorter pulses after recompression stage are expected to be generated in the copropragating amplification scheme. However, a better optical quality of the compressed pulses with lower substructures is achieved in backward configuration. 


\section{Acknowledgment}

The author would like to thank Guy Millot, Stéphane Pitois and Stefano Wabnitz for fruitful discussions.

This work has been supported by the Fond National pour la Science under contract "ACIPhotonique PH43", by the Centre National de la Recherche Scientifique, and by the Conseil Régional de Bourgogne. 


\section{REFERENCES}

[1] M.E Fermann, V.I. Kruglov, B.C.Thomsen, J.M. Dudley, J.D. Harvey, Phys. Rev. Lett. 84 (2000) 6010.

[2] C. Finot, G. Millot, J.M. Dudley, Opt. Lett., 29 (2004) 2533.

[3] V.I. Kruglov, A.C. Peacock, J.D. Harvey, J.M. Dudley, J. Opt. Soc. Am. B 19 (2002) 461.

[4] D. Anderson, M. Desaix, M. Karlson, M.Lisak and M.L. Quiroga-Teixeiro, J. Opt. Soc. Am. B 10 (1993), 1185.

[5] A. Malinowski, A. Piper, J.H.V Price, K. Furusawa, Y. Jeong, J. Nilsson, D.J. Richardson, Opt. Lett., 29 (2004), 2073.

[6] J. Limpert, T. Schreiber, T. Clausnitzer, K. Zöllner, H.-J. Fuchs, E.-B Bley, H. Zellmer and A. Tünnermann, Opt. Express, 10, (2002), 628.

[7] K. Tamura, N. Nakazawa, Opt Lett. 21, (1996) 68.

[8] F. Ö Ilday, J.R. Buckley, W.G. Clark and F.W. Wise, Phys. Rev. Lett. 92, (2004) 213902.

[9] C. Finot, G. Millot, Optics Express, 12 (2004), 5104

[10] Y. Ozeki, Y. Takushima, K. Taira, K. Kikuchi, in Conference on Lasers and ElectroOptics (CLEO) 2004, CTuK2.

[11] C. Finot, G. Millot, C. Billet, J.M. Dudley, Opt. Express 11 (2003), 1547.

[12] V.I. Kruglov, A.C. Peacock, J.M. Dudley, J.D Harvey, Opt. Lett., 25 (2000), 1753.

[13] S. Boscolo, S.K. Turitsyn, V.Yu. Novokshenov, J.H.B. Nijhof, Theor. Math. Phys., 133 (2002), 1647.

[14] C. Finot, G. Millot, S. Pitois, C. Billet, J-M Dudley, IEEE J. Sel. Topics Quantum Electron., 10 (2004), 1211.

[15] R. Trebino, Frequency Resolved Optical Gating. The Measurement of Ultrashort Laser Pulsers, Kluwer Academic Publishers (2000).

[16] G. P. Agrawal, Nonlinear Fiber Optics, Third Edition, Academic Press, San Francisco, 2001.

[17] R. H. Stolen, J.P. Gordon, W.J. Tomlinson and H.A. Haus, J. Opt. Soc. Am. B. 6, (1989), 1159.

[18] B. Min, W. J. Lee, N. Park, IEEE Photon. Technol. Lett. 12 (2000) 1486.

[19] Q. Lin and G.P. Agrawal, J. Opt. Soc. Am. B 20 (2003) 1616. 


\section{FIGURE CAPTIONS}

Fig 1. Numerical study of the influence of the amplification scheme for different configurations : backward configuration (curve A, solid line), forward configuration (curve B, dotted line) and constant gain (curve C, dashed line). (a) Evolution of the FWHM temporal width of the pulse during the propagation in the $5.3 \mathrm{~km}$ Raman amplifier. Results based on the resolution of the set of coupled NLSE (2) for co and counter propagating configuration are displayed by circles. They are compared with results based on the use of the standard NLSE with longitudinally varying gain (4) (solid, dashed and dotted lines) and with analytical asymptotic predictions (diamonds). (b) Gain evolution along the fiber for different configurations.

Fig 2. Schematic diagrams of experimental setups (a) Forward configuration (b) Backward configuration

Fig 3. Intensity and chirp profiles. Results of output pulse FROG trace retrievals are displayed with open circles (backward pumping) and crosses (forward pumping). The experimental results are compared with numerical simulations (respectively solid and dashed lines for counter- and co-propagating configurations). For the clarity of the figure, chirp profiles have been downshifted (backward configuration) or upshifted (forward configuration). The input pulse is represented by diamonds with a magnification factor of 20 . (a) Results with an initial pulse energy of $1.5 \mathrm{pJ}$ (b) Results with an initial pulse energy of 3 $\mathrm{pJ}$

Fig 4. Influence of the initial pulse energy on the output pulse temporal width and on the power required to achieve a $20 \mathrm{~dB}$ integrated gain. Experimental results are displayed by circles (backward configuration) and crosses(forward configuration) and are compared with simulations (respectively solid and dotted lines). Simulations results based on a constant gain approximation are represented by a dashed line. (a) Evolution of the FWHM temporal width of the autocorrelation function of the amplified pulse versus the initial input pulse energy. (b) Evolution of the pump power required to obtain a $20 \mathrm{~dB}$ integrated gain versus the initial input pulse energy. 

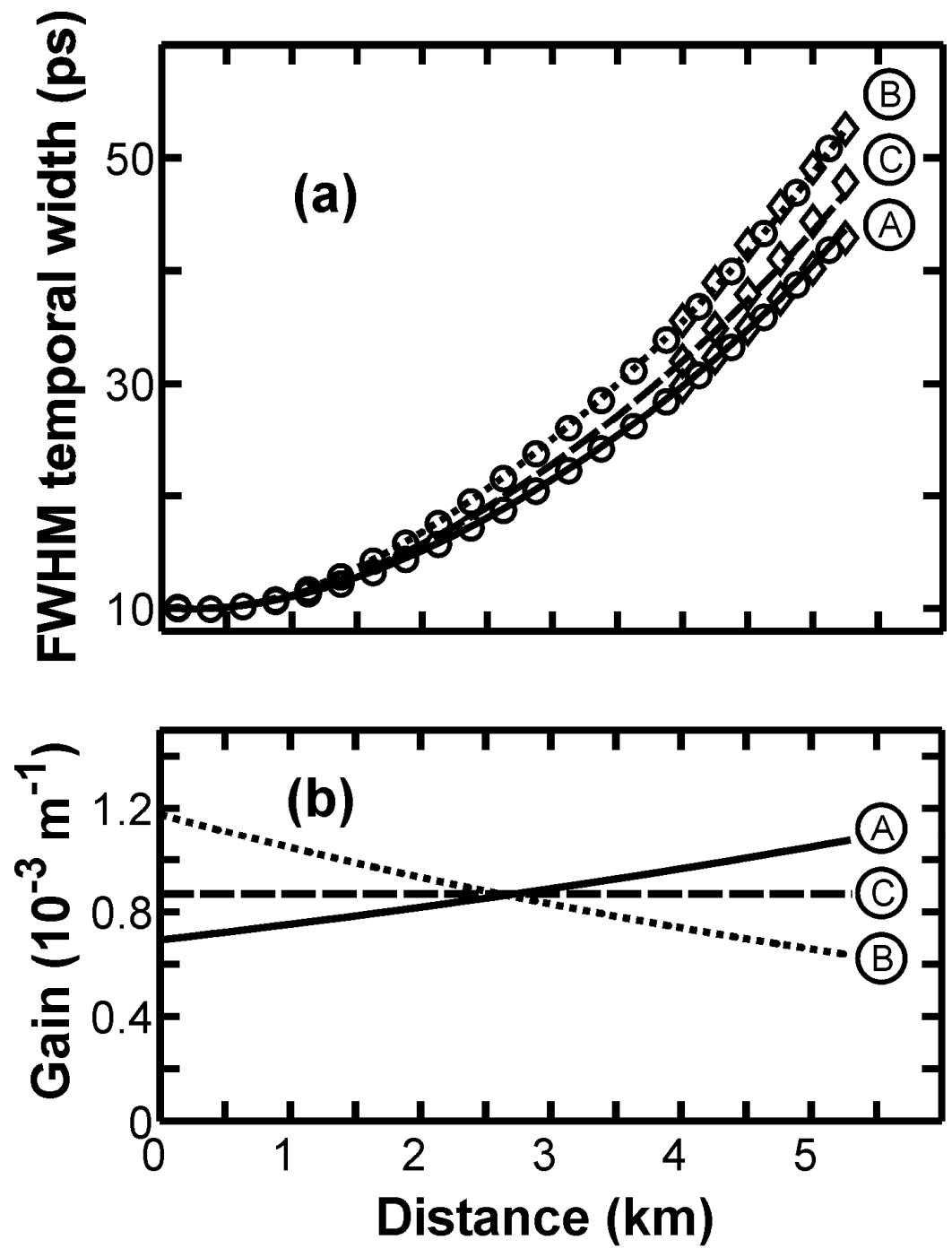

Figure 1 


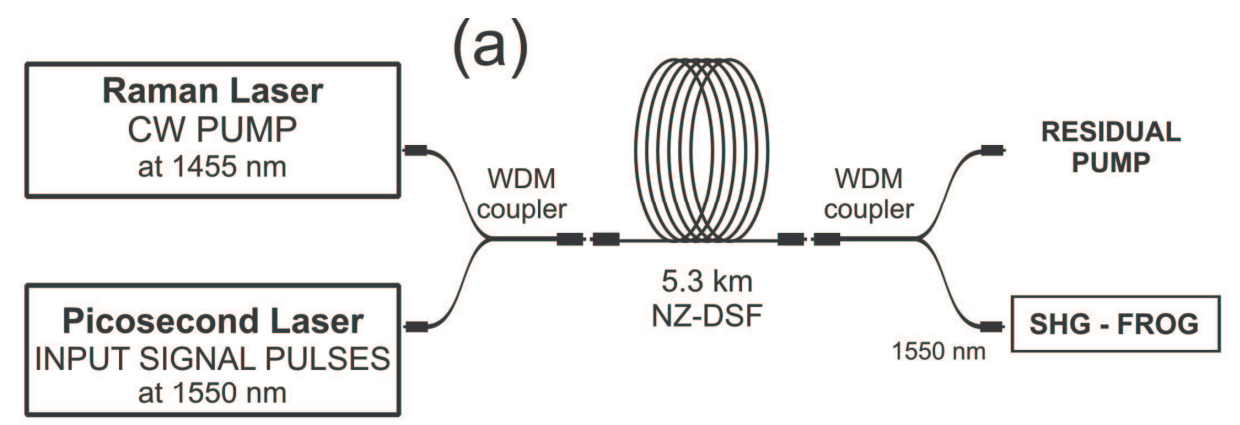

(b)

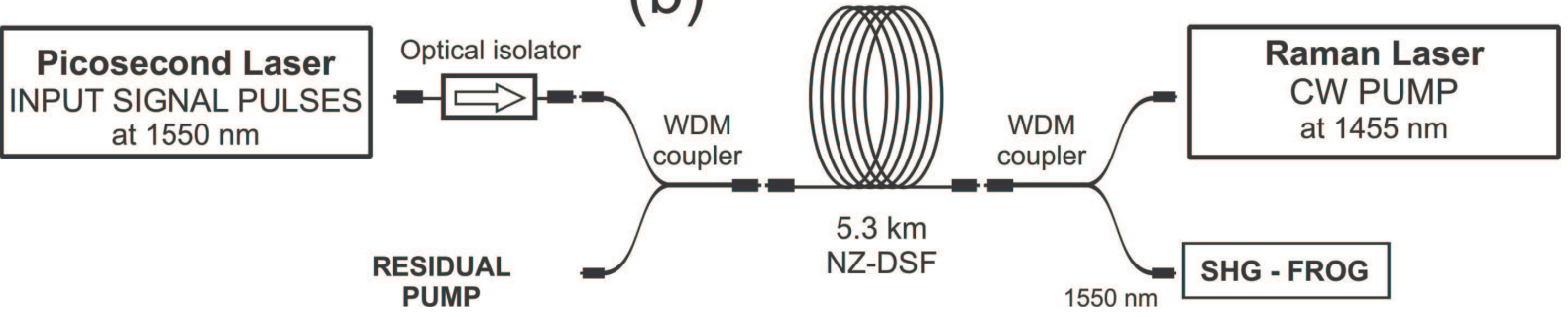

Figure 2 

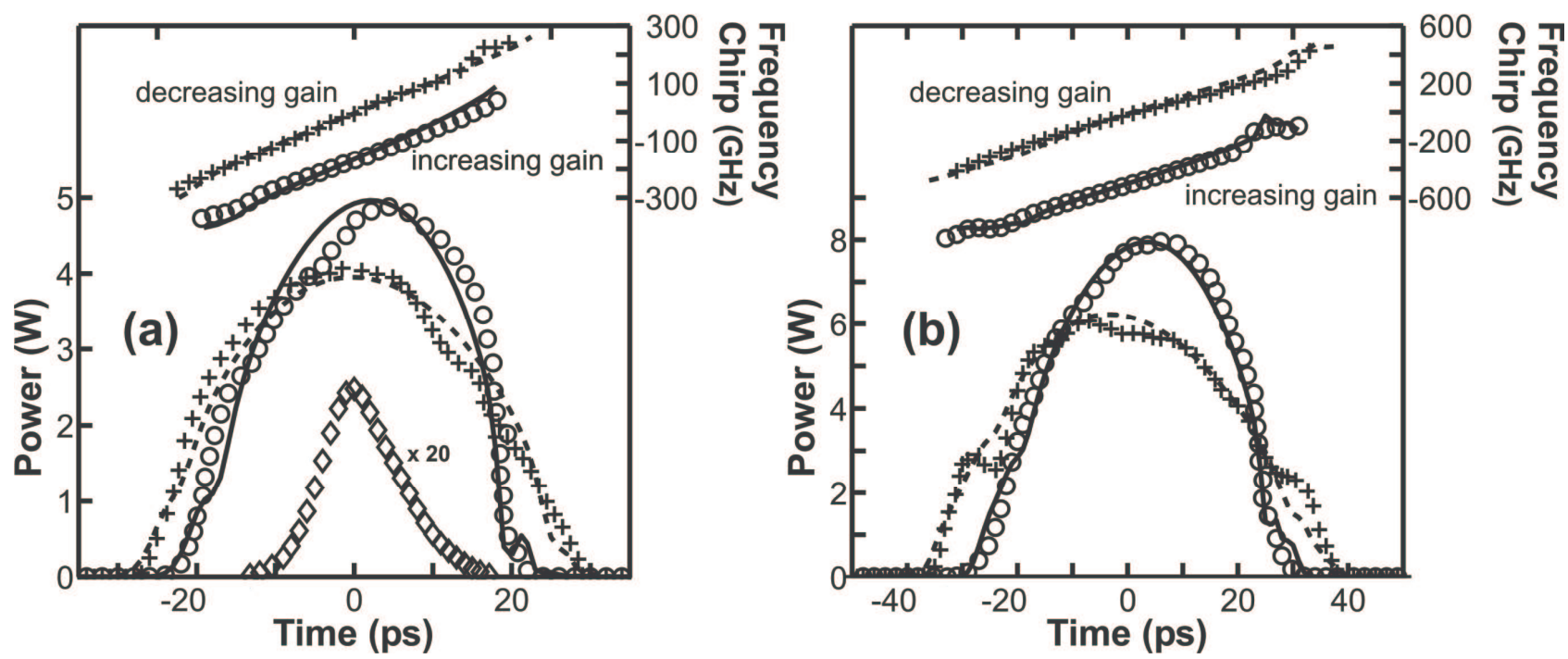

Figure 3 

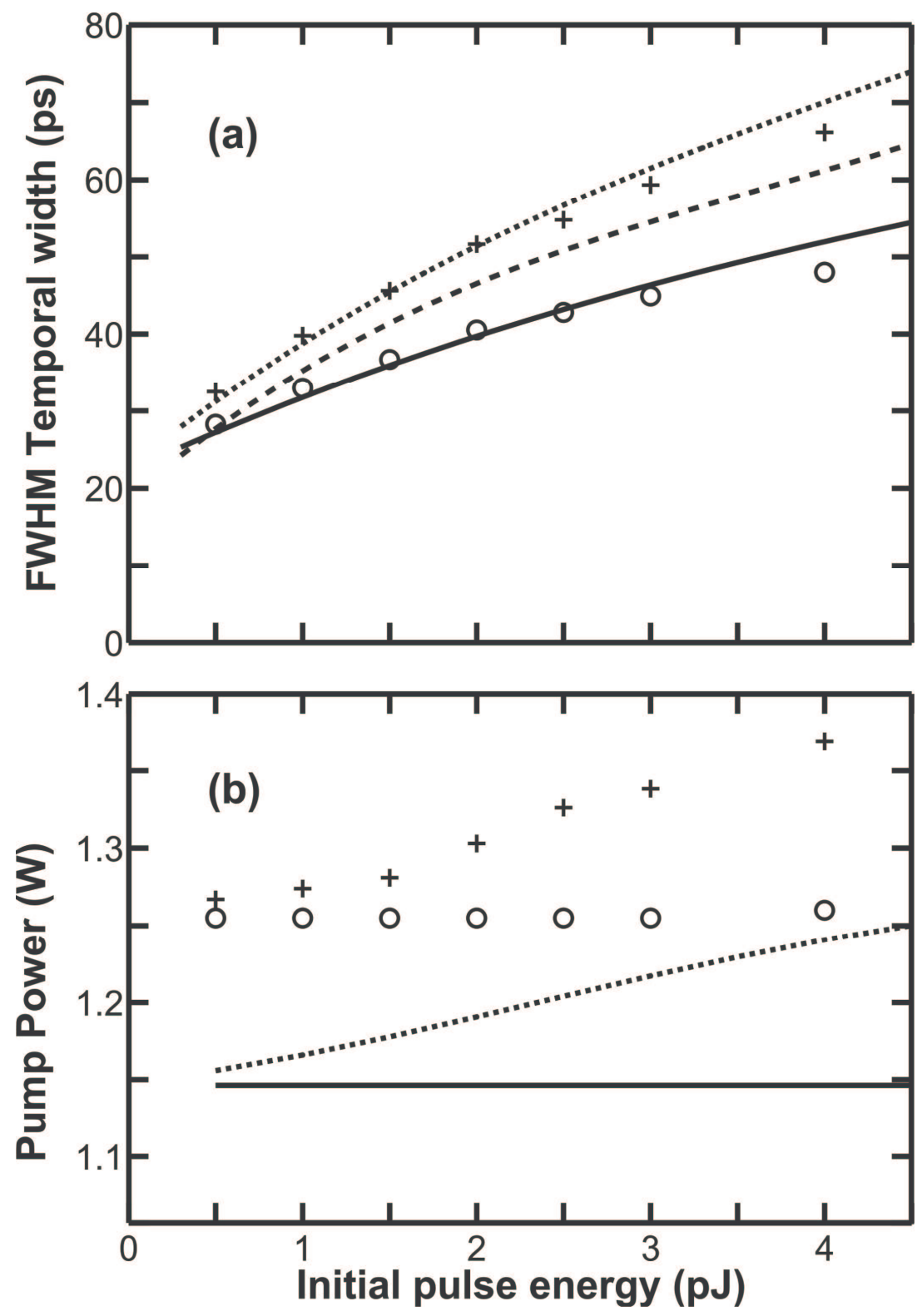

Figure 4 